\title{
Spotlight on postural control in patients with multiple sclerosis
}

This article was published in the following Dove Press journal: Degenerative Neurological and Neuromuscular Disease

\section{Luca Prosperini ${ }^{1}$ \\ Letizia Castelli²}

'Department of Neurosciences, San Camillo-Forlanini Hospital, Rome, Italy; ${ }^{2}$ Department of Neurology and Psychiatry, Sapienza University, Rome, Italy
Correspondence: Luca Prosperini Department of Neurosciences, San Camillo-Forlanini Hospital, C.ne Gianicolense 87, 00152 Rome, Italy $\mathrm{Tel}+39658704349$

Fax +3965870 4206

Email luca.prosperini@gmail.com

\begin{abstract}
Multiple sclerosis (MS) is a disease that heavily affects postural control, predisposing patients to accidental falls and fall-related injuries, with a relevant burden on their families, health care systems and themselves. Clinical scales aimed to assess balance are easy to administer in daily clinical setting, but suffer from several limitations including their variable execution, subjective judgment in the scoring system, poor performance in identifying patients at higher risk of falls, and statistical concerns mainly related to distribution of their scores. Today we are able to objectively and reliably assess postural control not only with laboratory-grade standard force platform, but also with low-cost systems based on commercial devices that provide acceptable comparability to gold-standard equipment. The sensitivity of measurements derived from force platforms is such that we can detect balance abnormalities even in minimally impaired patients and predict the risk of future accidental falls accurately. By manipulating sensory inputs (dynamic posturography) or by adding a concurrent cognitive task (dual-task paradigm) to the standard postural assessment, we can unmask postural control deficit even in patients at first demyelinating event or in those with a radiologic isolated syndrome. Studies on neuroanatomical correlates support the multifactorial etiology of postural control deficit in MS, with the association with balance impairment being correlated with cerebellum, spinal cord, and highly ordered processing network according to different studies. Postural control deficit can be managed by means of rehabilitation, which is the most important way to improve balance in patients with MS, but there are also suggestions of a beneficial effect of some pharmacologic interventions. On the other hand, it would be useful to pay attention to some drugs that are currently used to manage other symptoms in daily clinical setting because they can further impair postural controls of patients with MS.
\end{abstract}

Keywords: multiple sclerosis, postural control, balance, accidental falls, force platform, cognitive-motor interference

\section{Introduction}

Postural control can be defined as the ability to uphold the body's center of gravity within the base of support while maintaining a specified posture, making a voluntary movement or reacting to an external disturbance. ${ }^{1}$

Postural control strategies include either compensatory postural adjustments following an unpredicted disturbance or anticipatory postural adjustments that counteract any destabilizing consequences of voluntary movement or predicted disturbance. ${ }^{2}$

Postural responses cannot be considered as "reflex-like" automatic response, but rather balance control represents a complex task that is controlled by a combination of central and peripheral components including spinal reflexes, supraspinal commands, 
and the integration of afferent and/or efferent signals passing through the visual, vestibular and somatosensory systems, respectively, by the central nervous system (CNS). ${ }^{3}$ Biometric factors, physiologic functions, cognitive processing, emotional status, visual feedback and cerebellar activity have shown to influence postural sway. Consequently, numerous disorders including injuries, aging or neurologic, otologic and orthopedic pathologies can adversely affect postural sway. Deficiency in any one of the mechanisms involved in postural control can produce detrimental effects on balance, causing a sense of instability, vulnerability, as well as predisposing to accidental falls and further injury. ${ }^{4}$

Multiple sclerosis (MS) is the most common cause of nontraumatic, progressive disability in young adults. ${ }^{5} \mathrm{MS}$ is both an inflammatory and neurodegenerative disease characterized by demyelination and several degrees of axonal loss of the brain and spinal cord. ${ }^{6}$ By affecting the ability of neurons to communicate with each other effectively, MS can also be depicted as a "disconnection syndrome" leading to a variety of neurologic and neuropsychological deficit. ${ }^{7}$ The deficient integration of neural pathways, due to the widespread and variable distribution of CNS damage in patients with MS, can also affect postural control and the ability to maintain adequate balance. ${ }^{8}$ Postural control can even be impaired by MS-driven damage to peripheral organs providing sensory inputs to CNS or involved in motor output (Figure 1).
Indeed, impaired postural control is often observed in MS, with approximately two-thirds of these patients reporting lack of balance and coordination as the main symptom affecting their mobility in daily living. ${ }^{9}$ Balance deficit reduces mobility and independence, leads to falls and injuries, and impacts upon overall quality of life. ${ }^{10}$ Fatigue, muscle weakness and spasticity further contribute to compromise adequate balance and predispose patients to accidental falls (Figure 2)..$^{8,11-14}$

\section{Accidental falls}

Overall, frequency of accidental falls in people affected by MS is greater than in general population, as indirectly suggested by case-control studies documenting an approximately twofold increased risk of fall-related fractures and/or injuries in patients with MS compared with sex/age-matched individuals without MS (Table 1). ${ }^{15-20}$

Observational studies conducted on young and middleaged MS sample also showed that the proportion of fallers may range from $30 \%$ to $63 \%$ in a time frame of $1-12$ months, with about $29 \%-45 \%$ being recurrent fallers. ${ }^{14}$ These proportions are quite impressive if we consider that about $30 \%$ of community-dwelling healthy adults over 65 years fall in a 12 -month period, and only $10 \%$ are recurrent fallers. ${ }^{21}$ Furthermore, the number of accidental falls was collected retrospectively in most of these observational studies, thus leading to an underestimation of events occurred. In

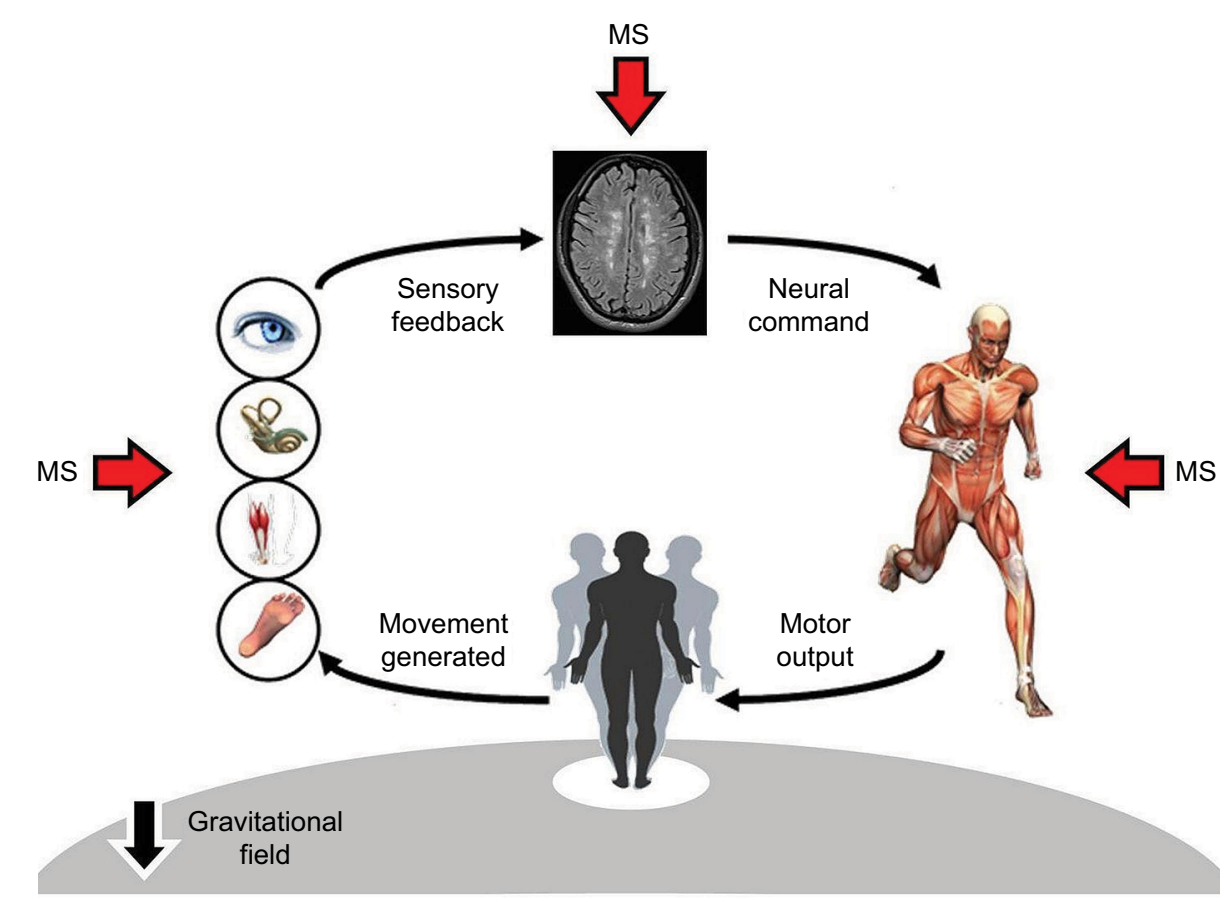

Figure I Schematic of postural control and its alterations in MS.

Abbreviation: MS, multiple sclerosis. 


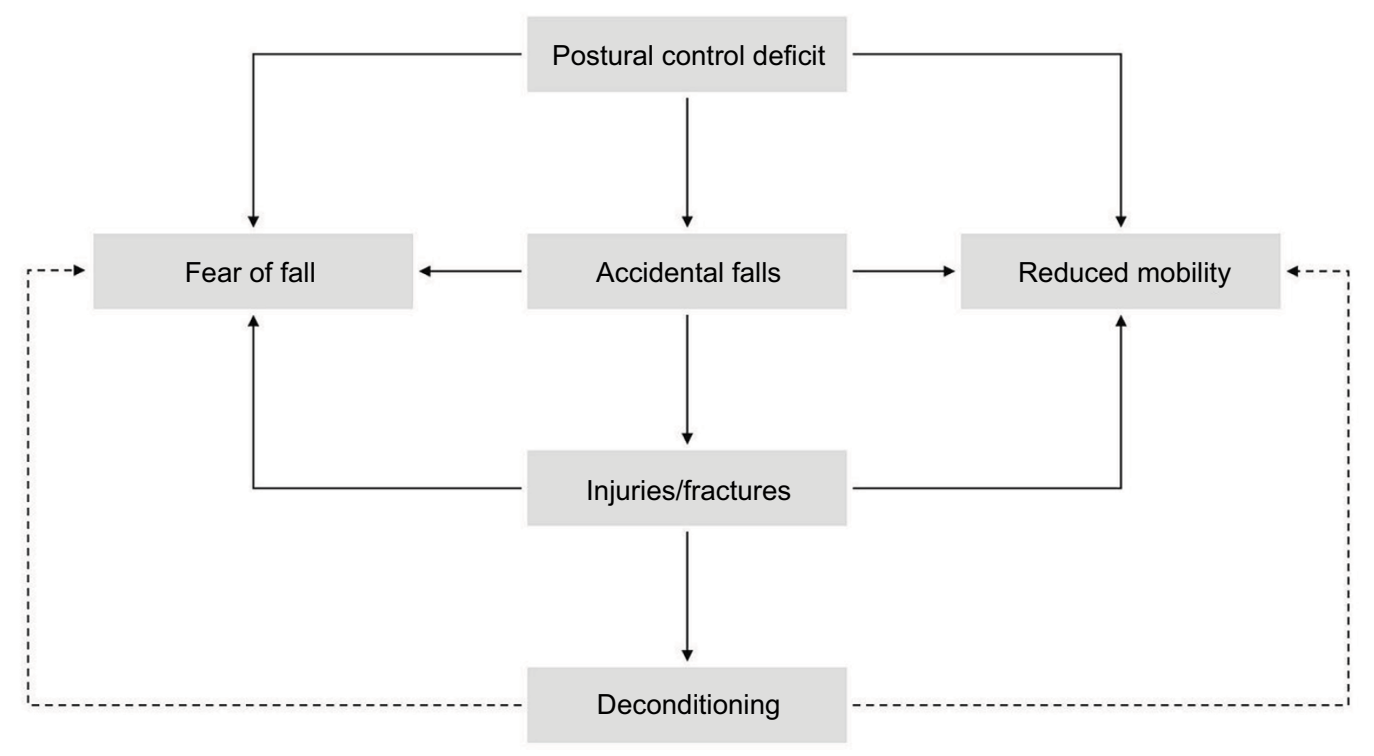

Figure 2 Consequences of postural control deficit in multiple sclerosis.

Table I Case-control studies exploring the incidence of fall-related fractures and injuries in patients with multiple sclerosis

\begin{tabular}{|c|c|c|c|c|c|}
\hline Study & Average age (years) & Cases & Controls & Outcome & Risk $^{\mathbf{a}}$ \\
\hline \multirow[t]{3}{*}{ Bazelier et al ${ }^{15}$} & 44.8 & 5,565 & 33,360 & Any fracture & 1.2 \\
\hline & & & & Hip fractures & 2.8 \\
\hline & & & & Osteoporotic fractures & 1.4 \\
\hline Cameron et $\mathrm{al}^{16}$ & 60.3 & 721 & 194,696 & Injurious falls & 1.9 \\
\hline \multirow[t]{2}{*}{ Bazelier et al ${ }^{17}$} & 43.6 & 2,415 & $|2,64|$ & Any fracture & 0.8 \\
\hline & & & & Fall-related fractures & 2.1 \\
\hline \multirow[t]{2}{*}{ Bazelier et al ${ }^{18}$} & 36.9 & 2,963 & 15,436 & Any fracture & 1.0 \\
\hline & & & & Femur/hip fractures & 1.9 \\
\hline \multirow[t]{2}{*}{ Ramagopalan et al ${ }^{19}$} & $\sim 50$ & 87,873 & $7,820,697$ & Any fracture & 1.9 \\
\hline & & & & Femoral fractures & 2.8 \\
\hline Bhattacharya et $\mathrm{al}^{20}$ & $\sim 65$ & 2,631 & $\mathrm{I}, 063,773$ & Hip fractures & 2.2 \\
\hline
\end{tabular}

Notes: ${ }^{\mathrm{V}}$ alues $>\mathrm{I}$ indicate increased risk in patients with multiple sclerosis.

fact, although there is a good correlation $(\mathrm{r}=0.82)$ between prospective recording and retrospective self-reporting of accidental falls, retrospective data are prone to recall bias, mainly because only falls resulting in injury are more likely to be reported by patients. ${ }^{22}$

Fall tendency may occur early in the course of the disease, even before walking and balance impairment become clinically evident and even after the first demyelinating event. ${ }^{23}$ Near-falls (i.e., a trip or stumble) occur commonly, but are underreported by patients with MS, despite the fact that near-falls and accidental falls share similar circumstances surrounding fall events, such as transferring outside the home and tripping over an obstacle; however, accidental falls can happen even indoor, especially in the kitchen or bathroom..$^{24,25}$
Factors that predispose patients with MS to accidental falls are a higher level of disability, a progressive disease course, the use of assistive devices for ambulation, a worse postural control and an impaired cognition. ${ }^{13,14,26}$

\section{Assessment of postural control}

Evaluating the level of impairment in postural control is clinically relevant for several purposes, including early detection of subjects at risk of falling, understanding of underlying pathophysiology and objective documentation of therapeutic efficacy.

Balance deficit can be quantified by means of clinical scales and force platforms. Clinical scales to evaluate balance encompass physician-rated scales, stop-watch measures 
and even self-administered questionnaires. Although some clinical scales are easy and relatively quick to use, they are hampered by their variable execution and subjective judgment in the scoring system..$^{27,28}$ Moreover, when used to predict patients at risk of falls, clinical scales have poor performance in discriminating between fallers and nonfallers, mainly due to their low sensitivity (i.e., performance in detecting fallers) and moderate-good specificity (i.e., performance in detecting nonfallers). ${ }^{29}$ Other limits of clinical scales are their nonlinear distribution and floor or ceiling effect, especially when evaluating patients with subtle balance deficit (Table 2). Therefore, some authors suggest that, in clinical practice, multiple tests should be administered for accurately identifying fallers. ${ }^{11}$ Alternatively, the Balance Evaluation Systems Test (BESTest) ${ }^{30}$ exhibits very good accuracy in predicting accidental falls $(>90 \%),{ }^{31}$ but it is time-consuming and requires many tools for the assessment of six different aspects of human balance, namely biomechanical constraints, stability limits, postural responses, anticipatory postural adjustments, sensory orientation and stability in gait. ${ }^{30}$ The use of a shorter version (mini-BESTest), with only a 10-minute administration time, could be more applicable to daily clinical setting. ${ }^{32}$

To overcome all the aforementioned limitations of clinical scales, there has been an increased interest about computerbased and objective instrumented measurements of balance. Posturography is an umbrella term that indicates all the techniques used to quantify postural control in upright stance, in either static or dynamic conditions, by means of a force platform..$^{33}$ Force platforms are instruments that measure ground reaction forces generated by a body standing on or moving across them, to quantify biomechanical parameters of human balance control. Static posturography refers to the measurement of postural sway of the body's center of pressure (COP) during quiet standing on a fixed support surface, while dynamic posturography involves the use of induced balance perturbation, such as shifting the support surface, using an unstable support surface, moving the visual surround, applying stimuli to upper body parts or performing voluntary weight shift. ${ }^{33}$

Static posturography provides objective measurements of postural control that have been shown to be not only highly reliable, but also valid in differentiating healthy controls from patients with MS. ${ }^{34-38}$ Furthermore, static standing balance measurements are more sensitive and accurate than a common clinical test (the Berg Balance Scale) in predicting accidental falls in MS.${ }^{35}$ This can be also displayed by the statokinesigram, that is, layout of a line connecting the successive positions of the body's COP during a stop-watch recording (Figure 3).

All posturographic techniques provide detailed and objective measurements of a wide range of time-domain or frequency-domain features of postural control, based on the sway of COP, that is, the point of application of the resultant from the vertical force's action. These measurements consist of many different parameters, including (but not limited to) speed, range, root mean square distance, path, area and 95\% confidence ellipse, and can be characterized on the anteroposterior or mediolateral axis or as the sum of the COP displacements. ${ }^{33}$

However, while static posturography is performed in conditions that are somewhat away from those encountered in daily-life activities, dynamic posturography seems to be a more ecologic approach to investigate postural control. Dynamic posturography assessment provides data on the motor and sensory contribution to balance control by manipulating one or more specific inputs (visual, vestibular or proprioceptive) involved in postural control..$^{39}$ Moreover,

Table 2 Clinical scales currently used to assess postural control and balance in multiple sclerosis

\begin{tabular}{|c|c|c|c|c|}
\hline Instrument & Brief description & $\begin{array}{l}\text { Time of } \\
\text { administration }\end{array}$ & $\begin{array}{l}\text { Overall } \\
\text { score }\end{array}$ & Direction \\
\hline Activities-Specific Balance Confidence & 16-item self-administered questionnaire & 15 minutes & $0-100$ & $\downarrow$ \\
\hline Balance Evaluation System Test & 36-item physician-rated scale & 30 minutes & $0-108$ & $\downarrow$ \\
\hline Berg Balance Scale & I4-item physician-rated scale & 15 minutes & $0-56$ & $\downarrow$ \\
\hline Dizziness Handicap Inventory & $\begin{array}{l}\text { Multidimensional } 25 \text {-item self- } \\
\text { administered questionnaire }\end{array}$ & 15 minutes & $0-100$ & $\uparrow$ \\
\hline Dynamic Gait Index & 8-item physician-rated scale & 10 minutes & $0-24$ & $\downarrow$ \\
\hline Falls Efficacy Scale International & 16-item self-reported questionnaire & 10 minutes & $0-100$ & $\uparrow$ \\
\hline Four-Square Step Test & Stop-watch measurement & $\leq 3$ minutes & N/A & $\uparrow$ \\
\hline Mini-BES Test & I4-item physician-rated scale & $10-15$ minutes & $0-28$ & $\downarrow$ \\
\hline Timed Up-and-Go Test & Stop-watch measurement & $\leq 3$ minutes & N/A & $\uparrow$ \\
\hline Tinetti Performance-Oriented Mobility & 16-item physician-rated scale & $10-15$ minutes & $0-48$ & $\downarrow$ \\
\hline Assessment & & & & \\
\hline
\end{tabular}

Note: Worse postural control indicated by lower $(\downarrow)$ or higher $(\uparrow)$ scores.

Abbreviation: N/A, not applicable. 


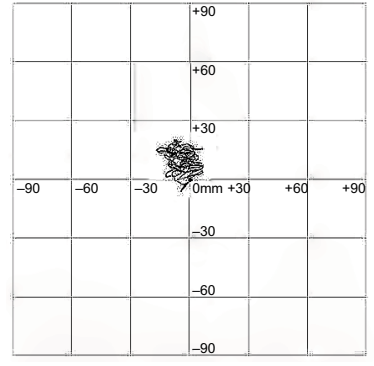

Healthy subject

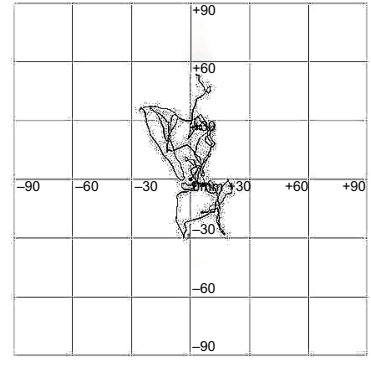

"Non-faller" subject with MS

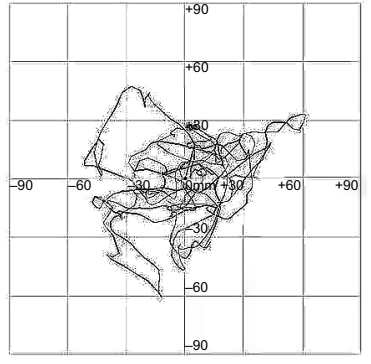

"Faller" subject with MS

Figure 3 Statokinesigrams collected by one healthy volunteer, one patient who did not report any fall and one patient who reported three accidental falls within the last 3 months.

Abbreviation: MS, multiple sclerosis.

these data can be combined into composite scores, such as the equilibrium score or the postural stability index. ${ }^{40}$ However, it has been found that $13 \%-22 \%$ of patients with MS fell under the more difficult conditions of dynamic posturography, especially when forced to rely only on a single input. ${ }^{41}$ Therefore, dynamic posturography seems to be less suitable than static posturography to assess postural control in patients with MS. Moreover, dynamic posturography requires an administration time longer than static posturography, and a more expensive and bulky equipment with respect to standard force platform.

Recently, low-cost force platforms using commercial devices have been proposed as a solution to overcome the aforementioned limitations of an expensive and bulky equipment. The commercial Wii balance board, an off-theshelf accessory used for playing with Wii and Wii U video game consoles (Nintendo, Kyoto, Japan), contains load cells detecting body's weight shifts. Although less accurate than standard force platforms because of their low-resolution measurements, low-cost systems based on Wii balance board can be useful in situations where lower accuracy and precision may be acceptable, as in daily clinical setting. ${ }^{42-44}$ While excellent test-retest reliability ( $>75 \%)$ was reported for Wii balance board-based low-cost systems, their agreement with standard force platform is at best adequate $(40 \%-74 \%){ }^{42}$ In fact, postural measurements obtained from Wii balance board are wider than those obtained from standard force platform, especially in conditions where there is lower performance variability (e.g., assessment of postural sway with eyes opened in subjects with mild disability). By contrast, Wii balance board performs more consistently with standard force platforms where there is higher performance variability (e.g., assessment of postural sway with eyes closed in subjects with greater disability). All these considerations imply that, from a clinical standpoint, low-cost systems can be effectively used in longitudinal study to test relative change in the same individual, while they are not recommendable to test absolute difference across individuals in cross-sectional study. Therefore, the clear advantage of these low-cost systems, representing a time-/space-/money-saving alternative to bulky and expensive laboratory-grade force platform, should be balanced with their disadvantages of a lower accuracy and worse signal-to-noise ratio.

Postural control can be reliably assessed also by the visual perception computing systems enabled with the Kinect camera, a motion-sensing input device developed for playing video games running on Xbox 360, Xbox One and Windowsequipped personal computers (Microsoft, Redmond, WA, USA). The Kinect system contains an RGB color Video Graphic Accelerator camera and an infrared depth sensor that work together to detect body motion and to display a real-time physical image. Other than as a surrogate for static posturography assessment, ${ }^{45}$ the Kinect has also been used as a surrogate for gait analysis in MS populations. ${ }^{46-48}$

\section{Cognitive-posture interference}

Patients with MS often exhibit deficits in both postural control and cognitive functions, and there is emerging evidence that a postural task and a cognitive task may interfere with each other when performed simultaneously ${ }^{49}$ raising the hypothesis of an overlap between network subserving the postural control and some cognitive functions. ${ }^{50,51}$ This phenomenon is generally defined as cognitive-motor interference and, in the specific case of balance, is called cognitive-posture interference. ${ }^{52}$

Cognitive-posture interference can be purposely investigated by means of dual-task paradigm experiments, that is, a study procedure that requires an individual to perform two tasks simultaneously. ${ }^{53}$ Ideally, a dual-task experiment should assess a cognitive task and a motor task without any mutual interference (single-task condition) and while performed concurrently (dual-task condition). This framework allows 
the estimation of the so-called dual-task cost, that is, the percentage change from single-task to dual-task condition, for the cognitive and the motor task. ${ }^{54}$

To better elucidate the relationship between the cognitive task and the motor task in dual-task situations, Plummer and Eskes ${ }^{54}$ proposed a graphical representation of the cognitivemotor interference patterns. This can be applied also to cognitive-postural interference phenomenon, by calculating the dual-task cost of balance and cognition according to the following formulas:

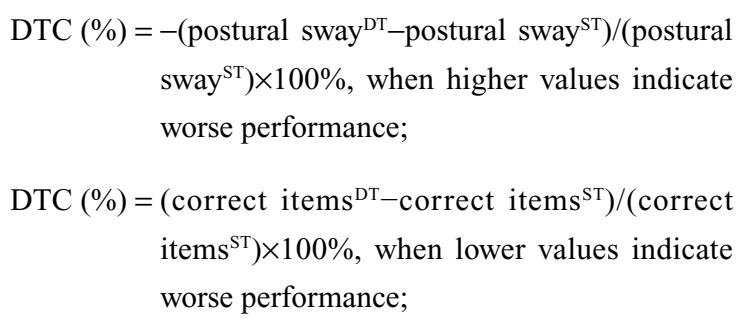

where DTC is dual-task cost, DT is dual-task, and ST is single-task.

Afterwards, the two dual-task costs, that of cognition and that of balance, can be plotted against each other to identify a specific pattern among nine possible patterns: mutual interference or facilitation, balance interference or facilitation, cognitive interference or facilitation, balance-priority or cognitivepriority tradeoff, and no interference (Figure 4). Worthy of note, no literature data and specific rules are currently available to define the boundaries of the no-interference area. ${ }^{54}$

Dual-task studies aimed at exploring the cognitivepostural interference phenomenon in MS populations were mainly based on static posturography assessment while performing a concomitant cognitive test. Most of these studies suggested a predominant pattern of balance interference, that is, the dual-task condition causes deterioration of postural control. ${ }^{49}$

However, the reciprocal effect of dual-tasking on the cognitive and a static balance task is not always explored in the literature, with the changes of cognitive performance during balance testing being reported only rarely. Another unsolved issue is which concurrent cognitive task has the most detrimental effect on postural control in patients with MS. Recently, it has been reported that cognitive taskexploring executive functions are the most suitable to unmask the cognitive-posture interference phenomenon, but these data require further confirmation. ${ }^{50}$

The clear advantage of dual-task paradigms is that they increase efficiently the sensitivity of a clinical test by exacerbating existing subtle deficits. Deterioration in balance when performed in conjunction with a concurrent cognitive task has been described indeed not only at the latest stage of MS, but also in patients without any overt cognitive deficit and even in subjects at the first demyelinating event or radiologically isolated syndrome, that is, asymptomatic subjects with neuroimaging findings suggestive of demyelinating disease. ${ }^{50,55,56}$ Several detrimental consequences of cognitivepostural interference have been reported in patients with MS, including an increased risk of accidental falls, a higher level of fatigue and a reduced quality of life, especially in terms of social function and physical role perception. ${ }^{57-60}$ Nonetheless, the clinical relevance of this phenomenon in MS populations needs to be investigated more deeply.

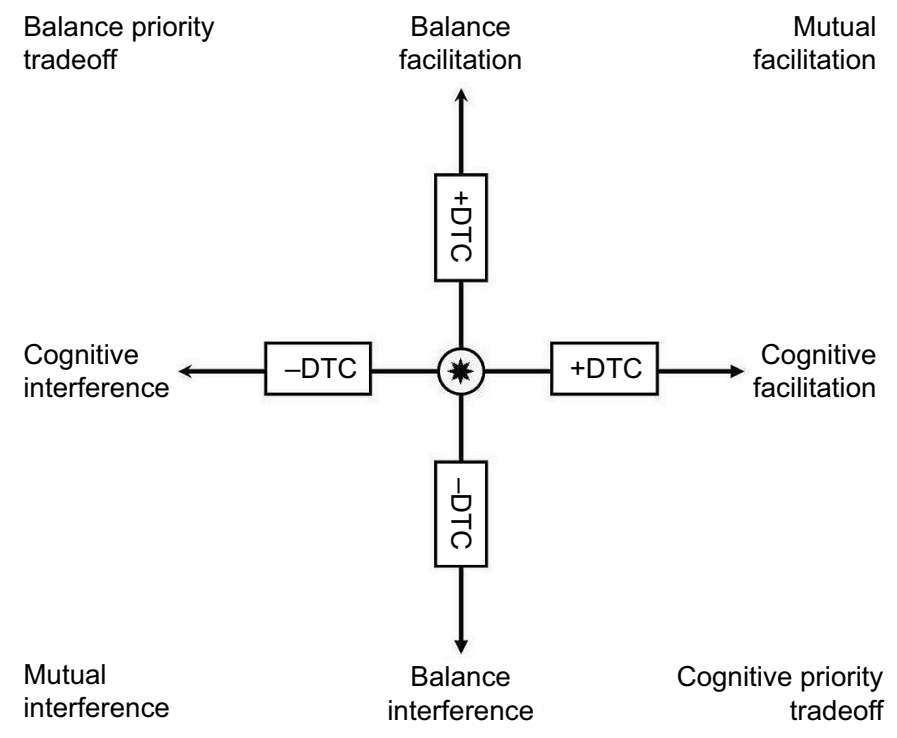

Figure 4 Patterns of cognitive-postural interference based on the reciprocal DTC of balance and cognition.

Note: The asterisk indicates no interference.

Abbreviation: DTC, dual-task cost. 


\section{Neuroanatomical correlates of postural control deficit}

The deficit of postural control observed in patients with MS, who have a widespread, variable and sometimes extensive damage into CNS, may be due to multifactorial causes that differ from person to person. Therefore, postural balance deficit has been generally interpreted as a result of an impaired central integration of visual, vestibular and somatosensory inputs. ${ }^{61}$ However, it is also possible that damage in specific locations of CNS is the primary contributor to MS-related balance deficit, as suggested by magnetic resonance imaging (MRI) studies.

One preliminary study found that the presence of a greater lesion volume (as seen on T2-weighted images) at brainstem level might be considered as a risk factor for multiple accidental falls (collected in a time frame of 6 months), regardless of the patient's disability level. ${ }^{62}$

Both slowed afferent proprioceptive conduction along demyelinated dorsal columns of spinal cord and damage of proprioceptive neural pathways originating from right brain hemisphere have been proposed as important causes of impaired postural control. ${ }^{63-65}$ Another hypothesis proposes the damage of cerebellar connections as the primary contributor to the balance impairment ${ }^{66}$ or, more extensively, the focal and diffuse involvement of the cerebellum, its connections, and other associative regions. ${ }^{67}$

The severity of balance impairment has been shown to be related with both damage along specific white matter tracts - including cerebellar peduncles, pons, thalamus and some supratentorial associative bundles - and regional atrophy of anterior lobules of the cerebellum (IV, V, VI) and lobule VIII. The remarkable disparity between the widespread abnormalities in white matter tracts and the selective grey matter damage of the cerebellum also suggests that postural control deficit is caused by a cerebellar atrophy secondary to disconnection from the cerebral cortex and the spinal cord. ${ }^{67}$

Clinically relevant association between impaired postural sway and damage of cerebellar connections has been demonstrated not only with conventional and nonconventional structural MRI, but also with resting-state functional MRI techniques. Compared to healthy subjects, patients with MS exhibit abnormalities of cortico-cerebellar circuits, with decreased connectivity in caudate nuclei and thalami, and increased connectivity in the cerebellum, pons, left amygdala and orbitofrontal cortices. In particular, decreased connectivity between dentate nuclei of cerebellum and the left caudate nucleus has been related with worse postural control. ${ }^{68}$ This altered connectivity within the cerebellar network suggests a compensatory mechanism leading to an adaptive role in maintaining an adequate balance, as also supported by a recent study showing an association between better postural control and greater connectivity within a specific area of the anterior lobe of cerebellum belonging to the leg motor network. ${ }^{69}$

However, networks involved in postural control regulation can be different in diverse situations. Consequently, damage at different levels of CNS can result in different types of balance deficit. When all sensory inputs (visual, proprioceptive and vestibular) are available, the resulting balance deficit is related mainly to cerebellum atrophy and demyelinating lesions along cerebellar connections, while spinal cord atrophy and demyelinating lesions in brainstem are the principal contributors of a worse postural control when the visual input is lacking. ${ }^{70}$

The severity of cognitive-posture interference due to MS has been related with a higher probability of detecting demyelinating lesions in distinct anatomical regions - namely the superior and anterior radiate - bilaterally. ${ }^{51}$ Therefore, it is possible that disconnection of brain circuitry between cerebellum, thalami, striatum and prefrontal areas - connected through the anterior and superior corona radiate - can impair the higher-level postural control required to maintain an adequate balance in dual-task situations. ${ }^{71}$

\section{Interventions to improve postural control}

Rehabilitation is currently the cornerstone for managing and improving postural control in patients with MS. There are a lot of strategies that have been proposed, including proprioceptive training, motor and sensory training, endurance training, step training, Pilates exercises, whole-body vibration, hippotherapy (equine-assisted therapy), balance-based torso weighting, Tai-Chi Chuan, vestibular rehabilitation, robot-assisted gait training, virtual reality and exergames. A recently published meta-analysis specifically addressing this topic revealed that gait and balance functional training interventions yielded the greatest effect on postural control, and showed that higher-intensity programs maximized effectiveness. ${ }^{72}$ Overall, these rehabilitative interventions promoted only a small decrease in fall risk, thus suggesting also that the magnitude of the improvements achieved was not sufficient to influence fall outcomes. ${ }^{72}$ However, the use of fall event as outcome measures is affected by several methodologic concerns, as discussed earlier (see also "Accidental falls" section). ${ }^{22}$ Furthermore, published studies suffer from a number of limitations that represent an avenue for future investigation, including small sample sizes, few data on long-term effect and retention of training-induced improvement, lack of standardized postural control outcomes, wide 
variability in terms of schedule, duration, frequency and intensity of rehabilitative interventions.

Postural control impairment cannot be effectively managed with pharmacologic interventions, because there is not yet any medication with indication for improving balance. Furthermore, some drugs that are broadly used in neurologic setting may even worsen gait and balance of patients with MS, including antidepressants (selective serotonin reuptake inhibitor and serotonin and norepinephrine reuptake inhibitor), centrally acting muscle relaxants, genitourinary medications and sex hormones (Table 3). ${ }^{73,74}$ If there is also a dose-dependent effect of the aforementioned classes of medications on the risk of accidental falls is still controversial. $22,75,76$

The potential effect of cannabinoids and its derivatives on postural control is still unclear. There are few case reports showing clinical improvement of motor coordination in patients with MS affected by ataxia. ${ }^{77,78}$ By contrast, it has also been reported that cannabis smoking further impairs posture and balance in patients with $\mathrm{MS}^{79}$ and, more recently, a large multicenter, postmarketing experience in Italy showed that drowsiness and dizziness are two relevant reasons for premature discontinuation of nabiximols, a plant-derived $\delta$-9-tetrahydrocannabinol-cannabidiol oromucosal spray medicine for add-on treatment of refractory MS-related spasticity. ${ }^{80}$ Furthermore, an independent study conducted on a small sample of patients with MS ( $n=22)$ has suggested a detrimental effect of nabiximols on postural control (detected at static posturography), especially in dual-task condition. ${ }^{81}$

Short-term improvement of postural control has been reported with prolonged-release oral dalfampridine, a voltage-gated potassium-channel blocker able to improve action potential conduction in demyelinated axons that was specifically licensed to improve walking in patients with MS..$^{82,83}$ More specifically, the beneficial effect of dalfampridine on balance might be mediated by an enhanced inhibitory drive and precision of pacemaking of cerebellar Purkinje cells. ${ }^{84}$

A potential efficacy of riluzole as symptomatic therapy in different forms of cerebellar ataxia, including two patients

Table 3 List of medications potentially affecting postural control in multiple sclerosis

\begin{tabular}{ll}
\hline Deteriorating postural control & Improving postural control \\
\hline Centrally acting muscle relaxants & Prolonged-release fampridine \\
Selective serotonin reuptake inhibitor & Riluzole \\
Serotonin-norepinephrine reuptake & Dopamine agonists \\
inhibitor & \\
Genitourinary compounds & \\
Cannabis & \\
Sexual hormones & \\
\hline
\end{tabular}

with MS, has been suggested in a double-blind randomized clinical trial. ${ }^{85}$ By opening small-conductance calciumactivated potassium channels, riluzole might reduce hyperexcitability of the neurons in the deep cerebellar nuclei that receive altered inhibitory input from Purkinje cells. ${ }^{86}$

Finally, the suggestive theory that dopamine agonists can reduce cognitive-posture interference has been recently raised, ${ }^{87}$ under the hypothesis that disconnection of CNS areas innervated by dopaminergic neurons (striatum and prefrontal cortex) is responsible for an increased dual-task cost of balance in patients with MS. ${ }^{51,88}$ However, to our knowledge, there are no data available to support the use of dopamine-agonist therapy in the MS setting.

\section{Conclusion}

Postural control is impaired early in the MS course, even in patients with minimal or no disability. Balance problems result in accidental falls and reduced mobility, negatively affecting quality of life and daily living activity.

Impaired postural control has multifactorial causes in MS, given the variable and extensive damage to the CNS. The role of rehabilitation in improving postural control deficit and, to a lesser extent, in reducing the risk of accidental falls is well established. At the moment there is no pharmacologic intervention specifically indicated for balance problems, but there are promising data favoring drugs that modulate neural transmission at cerebellar and prefrontal network level.

\section{Acknowledgment}

The authors wish to thank Ms. Eleonora Boni for the English language assistance.

\section{Disclosure}

LP received consulting fees, and/or lecture fees, and/or travel grants from Almirall, Biogen, Genzyme, Merck Serono, Novartis, Roche and Teva. LC received consulting fees and travel grants from Almirall. The authors report no other conflicts of interest in this work.

\section{References}

1. Pollock AS, Durward BR, Rowe PJ, Paul JP. What is balance? Clin Rehabil. 2000;14(4):402-406.

2. Massion J. Postural control systems in developmental perspective. Neurosci Biobehav Rev. 1998;22(4):465-472.

3. Marsden CD, Merton PA, Morton HB. Human postural responses. Brain J Neurol. 1981;104(3):513-534.

4. Fasano A, Plotnik M, Bove F, Berardelli A. The neurobiology of falls. Neurol Sci. 2012;33(6):1215-1223.

5. Compston A, Coles A. Multiple sclerosis. Lancet Lond Engl. 2008;372(9648):1502-1517. 
6. Matthews PM, Roncaroli F, Waldman A, et al. A practical review of the neuropathology and neuroimaging of multiple sclerosis. Pract Neurol. 2016;16(4):279-287.

7. Dineen RA, Vilisaar J, Hlinka J, et al. Disconnection as a mechanism for cognitive dysfunction in multiple sclerosis. Brain J Neurol. 2009;132(Pt 1):239-249.

8. Cameron MH, Lord S. Postural control in multiple sclerosis: implications for fall prevention. Curr Neurol Neurosci Rep. 2010;10(5):407-412.

9. Van Asch P. Impact of mobility impairment in multiple sclerosis 2-patients' perspectives. Eur Neurol Rev. 2011;6:115-120.

10. Peterson EW, Cho CC, Finlayson ML. Fear of falling and associated activity curtailment among middle aged and older adults with multiple sclerosis. Mult Scler. 2007;13(9):1168-1175.

11. NilsagårdY, Denison E, Gunnarsson L-G, Boström K. Factors perceived as being related to accidental falls by persons with multiple sclerosis. Disabil Rehabil. 2009;31(16):1301-1310.

12. Finlayson ML, Peterson EW, Cho CC. Risk factors for falling among people aged 45 to 90 years with multiple sclerosis. Arch Phys Med Rehabil. 2006;87(9):1274-1279; quiz 1287.

13. Gunn HJ, Newell P, Haas B, Marsden JF, Freeman JA. Identification of risk factors for falls in multiple sclerosis: a systematic review and meta-analysis. Phys Ther. 2013;93(4):504-513.

14. Giannì C, Prosperini L, Jonsdottir J, Cattaneo D. A systematic review of factors associated with accidental falls in people with multiple sclerosis: a meta-analytic approach. Clin Rehabil. 2014;28(7):704-716.

15. Bazelier MT, van Staa T, Uitdehaag BM, et al. The risk of fracture in patients with multiple sclerosis: the UK general practice research database. J Bone Miner Res. 2011;26(9):2271-2279.

16. Cameron MH, Poel AJ, Haselkorn JK, Linke A, Bourdette D. Falls requiring medical attention among veterans with multiple sclerosis: a cohort study. J Rehabil Res Dev. 2011;48(1):13-20.

17. Bazelier MT, van Staa TP, Uitdehaag BM, et al. Risk of fractures in patients with multiple sclerosis: a population-based cohort study. $\mathrm{Neu}$ rology. 2012;78(24):1967-1973.

18. Bazelier MT, Bentzen J, Vestergaard P, et al. The risk of fracture in incident multiple sclerosis patients: the Danish National Health Registers. Mult Scler. 2012;18(11):1609-1616.

19. Ramagopalan SV, Seminog O, Goldacre R, Goldacre MJ. Risk of fractures in patients with multiple sclerosis: record-linkage study. $B M C$ Neurol. 2012;12:135.

20. Bhattacharya RK, Vaishnav N, Dubinsky RM. Is there an increased risk of hip fracture in multiple sclerosis? Analysis of the Nationwide Inpatient Sample. J Multidiscip Healthc. 2014;7:119-122.

21. Gillespie LD, Robertson MC, Gillespie WJ, et al. Interventions for preventing falls in older people living in the community. Cochrane Database Syst Rev. 2009;(2):CD007146.

22. Nilsagård Y, Lundholm C, Denison E, Gunnarsson L-G. Predicting accidental falls in people with multiple sclerosis-a longitudinal study. Clin Rehabil. 2009;23(3):259-269.

23. Moen SM, Celius EG, Nordsletten L, Holmøy T. Fractures and falls in patients with newly diagnosed clinically isolated syndrome and multiple sclerosis. Acta Neurol Scand Suppl. 2011;(191):79-82.

24. Carling A, Forsberg A, Nilsagård Y. Falls in people with multiple sclerosis: experiences of 115 fall situations. Clin Rehabil. Epub 2017 Sep 1

25. Fritz NE, Eloyan A, Baynes M, et al. Distinguishing among multiple sclerosis fallers, near-fallers and non-fallers. Mult Scler Relat Disord. 2017;19:99-104.

26. D’Orio VL, Foley FW, Armentano F, et al. Cognitive and motor functioning in patients with multiple sclerosis: neuropsychological predictors of walking speed and falls. J Neurol Sci. 2012;316(1-2):42-46.

27. Mancini M, Horak FB. The relevance of clinical balance assessment tools to differentiate balance deficits. Eur J Phys Rehabil Med. 2010;46(2):239-248.

28. Prosperini L, Pozzilli C. The clinical relevance of force platform measures in multiple sclerosis: a review. Mult Scler Int. 2013;2013:756564.

29. Cattaneo D, Regola A, Meotti M. Validity of six balance disorders scales in persons with multiple sclerosis. Disabil Rehabil. 2006;28(12): 789-795.
30. Horak FB, Wrisley DM, Frank J. The Balance Evaluation Systems Test (BESTest) to differentiate balance deficits. Phys Ther. 2009;89(5): 484-498.

31. Jacobs JV, Kasser SL. Balance impairment in people with multiple sclerosis: preliminary evidence for the Balance Evaluation Systems Test. Gait Posture. 2012;36(3):414-418.

32. Ross E, Purtill H, Uszynski M, et al. Cohort study comparing the Berg balance scale and the mini-BESTest in people who have multiple sclerosis and are ambulatory. Phys Ther. 2016;96(9):1448-1455.

33. Visser JE, Carpenter MG, van der Kooij H, Bloem BR. The clinical utility of posturography. Clin Neurophysiol. 2008;119(11):2424-2436.

34. Sosnoff JJ, Socie MJ, Boes MK, et al. Mobility, balance and falls in persons with multiple sclerosis. PLoS One. 2011; 6(11):e28021.

35. Prosperini L, Fortuna D, Giannì C, Leonardi L, Pozzilli C. The diagnostic accuracy of static posturography in predicting accidental falls in people with multiple sclerosis. Neurorehabil Neural Repair. 2013;27(1):45-52.

36. Kalron A, Achiron A. Postural control, falls and fear of falling in people with multiple sclerosis without mobility aids. J Neurol Sci. 2013;335(1-2):186-190.

37. Wajda DA, Motl RW, Sosnoff JJ. Three-month test-retest reliability of center of pressure motion during standing balance in individuals with multiple sclerosis. Int J MS Care. 2016;18(2):59-62.

38. Reguera-García MM, de Souza-Teixeira F, Fernández JA de P. Test-retest reliability of static postural control in people with multiple sclerosis. J Phys Ther Sci. 2017;29(8):1399-1404.

39. Nashner LM, Shupert CL, Horak FB, Black FO. Organization of posture controls: an analysis of sensory and mechanical constraints. Prog Brain Res. 1989;80:411-418; discussion 395-397.

40. Chaudhry H, Findley T, Quigley KS, et al. Measures of postural stability. J Rehabil Res Dev. 2004;41(5):713-720.

41. Cattaneo D, Jonsdottir J. Sensory impairments in quiet standing in subjects with multiple sclerosis. Mult Scler. 2009;15(1):59-67.

42. Castelli L, Stocchi L, Patrignani M, Sellitto G, Giuliani M, Prosperini L. We-Measure: toward a low-cost portable posturography for patients with multiple sclerosis using the commercial Wii balance board. J Neurol Sci. 2015;359(1-2):440-444.

43. Severini G, Straudi S, Pavarelli C, et al. Use of Nintendo Wii Balance Board for posturographic analysis of Multiple Sclerosis patients with minimal balance impairment. J Neuroeng Rehabil. 2017;14(1):19.

44. Keune PM, Young WR, Paraskevopoulos IT, et al. Measuring standing balance in multiple sclerosis: further progress towards an automatic and reliable method in clinical practice. J Neurol Sci. 2017;379: 157-162.

45. Behrens JR, Mertens S, Krüger T, et al. Validity of visual perceptive computing for static posturography in patients with multiple sclerosis. Mult Scler. 2016;22(12):1596-1606.

46. Newland P, Wagner JM, Salter A, et al. Exploring the feasibility and acceptability of sensor monitoring of gait and falls in the homes of persons with multiple sclerosis. Gait Posture. 2016;49:277-282.

47. Otte K, Kayser B, Mansow-Model S, Thomas FP, Skubic M, Rantz M. Accuracy and reliability of the kinect version 2 for clinical measurement of motor function. PLoS One. 2016;11(11):e0166532.

48. Gholami F, Trojan DA, Kovecses J, Haddad WM, Gholami B. A microsoft kinect-based point-of-care gait assessment framework for multiple sclerosis patients. IEEE J Biomed Health Inform. 2017;21(5):1376-1385.

49. Wajda DA, Sosnoff JJ. Cognitive-motor interference in multiple sclerosis: a systematic review of evidence, correlates, and consequences. BioMed Res Int. 2015;2015:720856.

50. Prosperini L, Castelli L, De Luca F, Fabiano F, Ferrante I, De Giglio L. Task-dependent deterioration of balance underpinning cognitivepostural interference in MS. Neurology. 2016;87(11):1085-1092.

51. Ruggieri S, Fanelli F, Castelli L, Petsas N, De Giglio L, Prosperini L. Lesion symptom map of cognitive-postural interference in multiple sclerosis. Mult. Scler. Epub 2017 Mar 1.

52. Leone C, Patti F, Feys P. Measuring the cost of cognitive-motor dual tasking during walking in multiple sclerosis. Mult Scler. 2015; 21(2):123-131. 
53. Della Sala S, Baddeley A, Papagno C, Spinnler H. Dual-task paradigm: a means to examine the central executive. Ann NY Acad Sci. 1995;769: 161-171.

54. Plummer P, Eskes G. Measuring treatment effects on dual-task performance: a framework for research and clinical practice. Front Hum Neurosci. 2015;9:225.

55. Kalron A, Dvir Z, Achiron A. Effect of a cognitive task on postural control in patients with a clinically isolated syndrome suggestive of multiple sclerosis. Eur J Phys Rehabil Med. 2011;47(4):579-586.

56. Dattola V, Logiudice AL, Bonanno L, et al. Does the radiologically isolated syndrome exist? A dual-task cost pilot study. Neurol Sci. 2017;38(11): 2007-2013.

57. Jacobs JV, Kasser SL. Effects of dual tasking on the postural performance of people with and without multiple sclerosis: a pilot study. J Neurol. 2012;259(6):1166-1176.

58. Wajda DA, Motl RW, Sosnoff JJ. Correlates of dual task cost of standing balance in individuals with multiple sclerosis. Gait Posture. 2014;40(3):352-356.

59. Castelli L, De Luca F, Marchetti MR, Sellitto G, Fanelli F, Prosperini L. The dual task-cost of standing balance affects quality of life in mildly disabled MS people. Neurol. Sci. 2016;37(5):673-679.

60. Etemadi Y. Dual task cost of cognition is related to fall risk in patients with multiple sclerosis: a prospective study. Clin Rehabil. 2017;31(2): 278-284.

61. Jackson RT, Epstein CM, De l'Aune WR. Abnormalities in posturography and estimations of visual vertical and horizontal in multiple sclerosis. Am J Otol. 1995;16(1):88-93.

62. Prosperini L, Castelli L, Sellitto G et al. Conventional magnetic resonance imaging features associated with accidental falls in multiple sclerosis. Mult Scler. 2015;21(4):492.

63. Cameron MH, Horak FB, Herndon RR, Bourdette D. Imbalance in multiple sclerosis: a result of slowed spinal somatosensory conduction. Somatosens Mot Res. 2008; 25(2):113-122.

64. Zackowski KM, Smith SA, Reich DS, et al. Sensorimotor dysfunction in multiple sclerosis and column-specific magnetization transfer-imaging abnormalities in the spinal cord. Brain. 2009;132(Pt 5):1200-1209.

65. Fling BW, Dutta GG, Schlueter H, Cameron MH, Horak FB. Associations between proprioceptive neural pathway structural connectivity and balance in people with multiple sclerosis. Front Hum Neurosci. 2014;8:814.

66. Prosperini L, Kouleridou A, Petsas N, et al. The relationship between infratentorial lesions, balance deficit and accidental falls in multiple sclerosis. J Neurol Sci. 2011;304(1-2):55-60.

67. Prosperini L, Sbardella E, Raz E, et al. Multiple sclerosis: white and gray matter damage associated with balance deficit detected at static posturography. Radiology. 2013;268(1):181-189.

68. Tona F, De Giglio L, Petsas N, et al. Multiple sclerosis: the role of cerebellar dentate functional connectivity in balance deficits. Radiology. Epub 2017 Dec 22.

69. Fling BW, Gera Dutta G, Horak FB. Functional connectivity underlying postural motor adaptation in people with multiple sclerosis. Neuroimage Clin. 2015;8:281-289.

70. Prosperini L, Petsas N, Raz E, et al. Balance deficit with opened or closed eyes reveals involvement of different structures of the central nervous system in multiple sclerosis. Mult Scler. 2014;20(1):81-90.
71. Leone C, Feys P, Moumdjian L, D'Amico E, Zappia M, Patti F. Cognitive-motor dual-task interference: a systematic review of neural correlates. Neurosci Biobehav Rev. 2017;75:348-360.

72. Gunn H, Markevics S, Haas B, Marsden J, Freeman J. Systematic review: the effectiveness of interventions to reduce falls and improve balance in adults with multiple sclerosis. Arch Phys Med Rehabil. 2015;96(10):1898-1912.

73. Stolze H, Klebe S, Zechlin C, Baecker C, Friege L, Deuschl G. Falls in frequent neurological diseases-prevalence, risk factors and aetiology. J Neurol. 2004;251(1):79-84.

74. Comber L, Quinn G, McGuigan C, Galvin R, Coote S. Medication usage and falls in people with multiple sclerosis. Mult Scler. Epub 2017 Sep 1.

75. Gunn H, Creanor S, Haas B, Marsden J, Freeman J. Risk factors for falls in multiple sclerosis: an observational study. Mult Scler. 2013;19(14): 1913-1922.

76. Cameron MH, Karstens L, Hoang P, Bourdette D, Lord S. Medications are associated with falls in people with multiple sclerosis: a prospective cohort study. Int J MS Care. 2015;17(5):207-214.

77. Clifford DB. Tetrahydrocannabinol for tremor in multiple sclerosis. Ann Neurol. 1983;13(6):669-671.

78. Meinck HM, Schönle PW, Conrad B. Effect of cannabinoids on spasticity and ataxia in multiple sclerosis. J Neurol. 1989;236(2): $120-122$.

79. Greenberg HS, Werness SA, Pugh JE, Andrus RO, Anderson DJ, Domino EF. Short-term effects of smoking marijuana on balance in patients with multiple sclerosis and normal volunteers. Clin Pharmacol Ther. 1994;55(3):324-328.

80. Patti F, Messina S, Solaro C, et al. Efficacy and safety of cannabinoid oromucosal spray for multiple sclerosis spasticity. J Neurol Neurosurg Psychiatry. 2016;87(9):944-951.

81. Castelli L, Prosperini L, Pozzilli C. Balance worsening associated with nabiximols in multiple sclerosis. Mult Scler. Epub 2018 Mar 1.

82. Prosperini L, Giannì C, Fortuna D, Marchetti MR, Pozzilli C. Oral dalfampridine improves standing balance detected at static posturography in multiple sclerosis. Mult Scler Int. 2014;2014:802307.

83. Hupperts R, Lycke J, Short C, et al. Prolonged-release fampridine and walking and balance in MS: randomised controlled MOBILE trial. Mult Scler. 2016;22(2):212-221.

84. Alviña K, Khodakhah K. The therapeutic mode of action of 4-aminopyridine in cerebellar ataxia. $J$ Neurosci. 2010;30(21):7258-7268.

85. Ristori G, Romano S, Visconti A, et al. Riluzole in cerebellar ataxia: a randomized, double-blind, placebo-controlled pilot trial. Neurology. 2010;74(10):839-845.

86. Raman IM, Gustafson AE, Padgett D. Ionic currents and spontaneous firing in neurons isolated from the cerebellar nuclei. $J$ Neurosci. 2000;20(24):9004-9016.

87. Yildiz A, Chmielewski W, Beste C. Dual-task performance is differentially modulated by rewards and punishments. Behav Brain Res. 2013;250:304-307.

88. Dobryakova E, Genova HM, DeLuca J, Wylie GR. The dopamine imbalance hypothesis of fatigue in multiple sclerosis and other neurological disorders. Front Neurol. 2015;6:52.
Degenerative Neurological and Neuromuscular Disease

\section{Publish your work in this journal}

Degenerative Neurological and Neuromuscular Disease is an international, peer-reviewed, open access journal focusing on research into degenerative neurological and neuromuscular disease, identification of therapeutic targets and the optimal use of preventative and integrated treatment interventions to achieve improved outcomes, enhanced

\section{Dovepress}

survival and quality of life for the patient. The manuscript management system is completely online and includes a very quick and fair peer-review system. Visit http://www.dovepress.com/testimonials.php to read real quotes from published authors. 